\title{
Excitation of metal casing using bent and folded dipole antenna with three conductors
}

\author{
Takumi Nishime ${ }^{1}$, Hiroshi Hashiguchi ${ }^{1}$, Naobumi Michishita ${ }^{1,}$ a), \\ and Hisashi Morishita ${ }^{1}$ \\ ${ }^{1}$ Graduate School of Science and Engineering, National Defense Academy \\ 1-10-20 Hashirimizu, Yokosuka, Kanagawa, 239-8686, Japan \\ a)naobumi@nda.ac.jp
}

Abstract: The antennas installed in mobile devices are small and lowprofile compared to wavelength at the operating frequency. In general, lowprofile horizontal elements and short monopole antennas have low radiation resistance. Low radiation resistance requires matching circuit between 50 $\Omega$ feed line and decreases radiation efficiency. By using the characteristic mode, excitation of the ground plane to improve the radiation resistance has been investigated. This paper proposes the structure with the modified folded dipole antenna, which is placed close to the metal casing. The surface current on the casing is excited by modified folded dipole antenna and contributes to the radiation.

Keywords: radiation efficiency, characteristic mode analysis (CMA), folded dipole antenna, 3D-platform-mounted antenna

Classification: Antennas and Propagation

\section{References}

[1] T. Beni, K. Kawabata, T. Thi Lan, and H. Arai, "Application of characteristic modes to design the platform-mounted inverted-F antenna," IEICE Commun. Express, vol. 9, no. 6, pp. 159-163, June 2020. DOI: 10.1587/comex.2019spl0006

[2] P.W. Futter and U. Jakobus, "LTE antenna design for tablet computers using characteristic mode analysis," $14^{\text {th }}$ Eur. Conf. Antennas Propag., Krakow, Poland, pp. 1-5, March 2020.

[3] R. Martens, E. Safin and D. Manteuffel, "Inductive and capacitive excitation of the characteristic modes of small terminals," 2011 Loughborough Antennas Propag. Conf., Loughborough, UK, pp. 1-4, Nov. 2011. DOI: 10.1109/lapc. 2011.6114141

[4] R. Harrington and J. Mautz, "Theory of characteristic modes for conducting bodies," IEEE Trans. Antennas Propag., vol. 19, no. 5, pp. 622-628, Sept. 1971. DOI: 10.1109/tap.1971.1139999

[5] T. Nishime, N. Michishita, and H. Morishita, "Characteristics modes on metal box excited by folded dipole antenna," IEICE Society Conf., B-1-62, Sept. 2020 (in Japanese).

[6] T. Nishio, K. Noguchi, K. Itoh, and J. Ida, "A high impedance folded dipole antenna with three conductors for energy harvesting applications," 2017 Int. Symp. Antennas Propag., Phuket, Thailand, pp. 1-2, Dec. 2017. 


\section{Introduction}

Cell phones, personal digital assistants, and other IoT devices use wireless communication, such as wireless local area network. These devices use inverted-F antennas, dielectric-loaded antennas, or small antennas for the miniaturization of a device. However, these miniaturized antennas have low radiation efficiency and are sensitive to the proximity of a conductor, such as a metal casing, circuit ground, or a human body. In general, the radiation resistance of a monopole antenna with an element length shorter than a quarter wavelength, or a horizontal element close to the ground plane is low, which makes it difficult to feed the antenna with $50 \Omega$ feed line. The low radiation resistance results in significantly decreased radiation efficiency. Excitation of the ground plane with small antennas has been considered for the utilization of the ground plane as the main radiator and to improve the radiation resistance. Characteristic mode analysis is a suitable method for determining the current distribution on a ground plane that behaves as an antenna [1, 2, 3, 4]. In previous studies $[1,2,3]$, an inductive coupling element (ICE), such as a small loop or slot antenna, is placed at current maxima, and a capacitive coupling element (CCE), such as a monopole antenna, is placed at current minima to excite current distribution. However, to maintain the radiation efficiency of the excitation element, a low-profile excitation element has not yet been considered. In addition, since the number of the excitation element was four in [1], the power divider is required and the feeding circuit becomes a complex. In this study, we propose a novel low-profile feeding structure with bent and folded dipole antenna with three conductors (BFDA) as a single excitation element for the metal casing with a similar size as the wavelength of the operating frequency. We verified the simulation results by measurement.

\section{Characteristic mode analysis of the metal casing with feeding structure}

\subsection{Feeding structure}

In ref [5], we analyzed the radiation from a casing fed by a folded dipole antenna. However, the reported antenna did not achieve impedance matching with the $50 \Omega$ feed line. In this study, we achieved impedance matching using a folded dipole antenna with three conductors [6]. Figure 1a shows the proposed BFDA designed at $2.45 \mathrm{GHz}$. The resonance frequency and input impedance of the antenna are optimized with $w_{3}, w_{1}, w_{2}, l_{1}, l_{2}$, and $l_{3}$. The parameters are $l_{1}=50.0 \mathrm{~mm}, l_{2}=23.0$ $\mathrm{mm}, l_{3}=1.0 \mathrm{~mm}, w_{1}=1.5 \mathrm{~mm}, w_{2}=2.5 \mathrm{~mm}$, and $w_{3}=2.0 \mathrm{~mm}$. Figure $1 \mathrm{~b}$ shows the current distribution of BFDA, which mounted apart on the infinite ground plane by $1.0 \mathrm{~mm}$. Then, the BFDA shows a radiation pattern similar to the end-fire array (y-direction) as shown in Fig. 1c. It is considered that although the radiation from the horizontal element is canceled out by the surface current of the ground plane, the folded part at approximately half-wavelength behaves as a monopole antenna with a phase difference of $180^{\circ}$ as shown in Fig. $1 \mathrm{~b}$. 


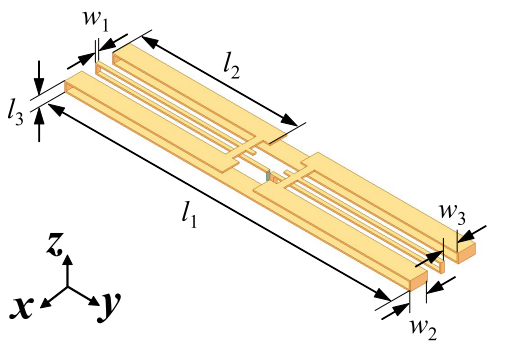

(a)

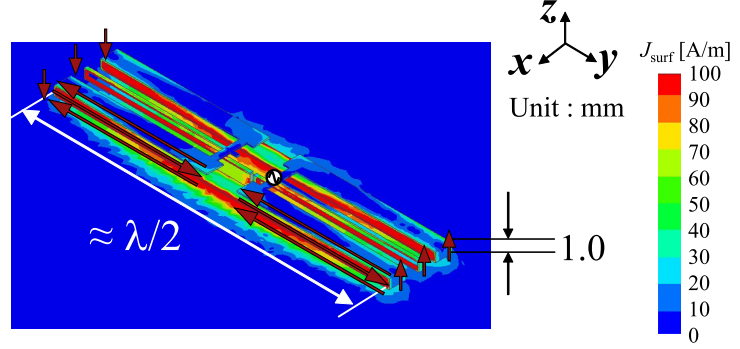

(b)

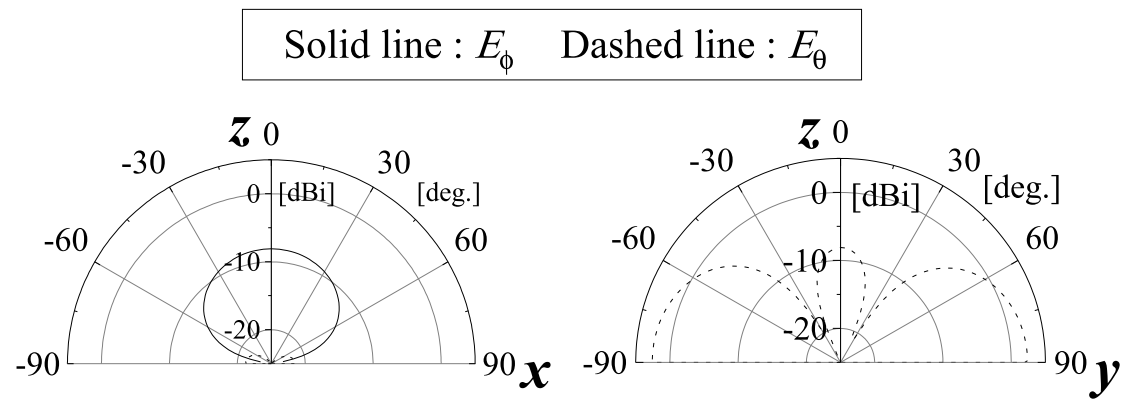

(c)

Fig. 1. (a) Dimension of BFDA. (b) Current distribution. (c) Radiation pattern on the infinite ground plane.

\subsection{Characteristic mode analysis}

In this section, the characteristic mode of a metal casing rectangular conductor of dimensions $100 \mathrm{~mm} \times 50 \mathrm{~mm} \times 23 \mathrm{~mm}$, as shown in Fig. 2a, is analyzed. The BFDA is mounted apart on the metal casing by $1.0 \mathrm{~mm}$. The size of the casing is determined based on the size of a common mobile device. Figure $2 b$ shows the modal weighting coefficient (MWC) of the BFDA mounted on the metal casing. In Fig. 2b, modes 2, and 5 are significantly excited by the BFDA. Figure $2 \mathrm{c}$ and Fig. 2d show the analysis results of the current distribution and radiation pattern of these characteristic modes $(\mathrm{CM})$ at $2.45 \mathrm{GHz}$. These modes show the BFDA does not contribute to the radiation itself. This is because the radiation from the horizontal element of BFDA is canceled out by the surface current of the metal casing. Then, the BFDA behaves as a feeding structure and the radiation is formed by the surface current of each $\mathrm{CM}$ of the metal casing excited by the BFDA. Therefore, the radiation from the BFDA with the metal casing is different from that without metal casing.

\section{Analysis and measurement result}

Figure 3a shows the fabricated antenna. A U.FL cable is installed inside and connected to an SMA connector to feed the BFDA. The hole for the cable installation is made on a small position of the current distribution of the major CM. The BFDA does not require a balun to connect with the coaxial line because the BFDA has a selfbalancing characteristic. Figure $3 \mathrm{~b}$ shows the reflection coefficient. The proposed antenna resonates at $2.45 \mathrm{GHz}$. Figure $3 \mathrm{c}$ shows the radiation pattern at $2.45 \mathrm{GHz}$. The radiation is mainly directed toward the $-z$-axis; the radiation toward the $+z$-axis, which is in front of the ground plane where the BFDA is placed, is $10 \mathrm{~dB}$ smaller than Received May 20, 2021 Accepted May 27, 2021 Publicized June 9, 2021 Copyedited September 1, 2021 


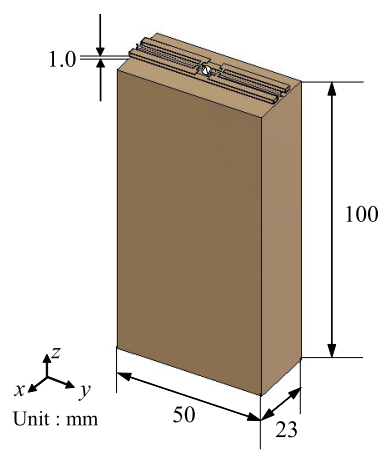

(a)

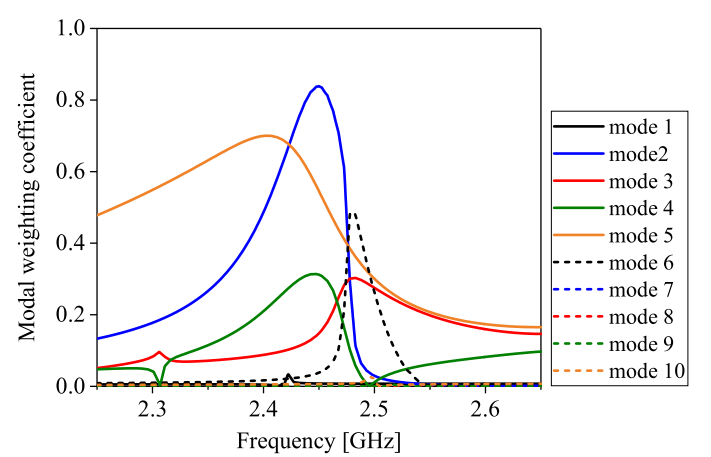

(b)

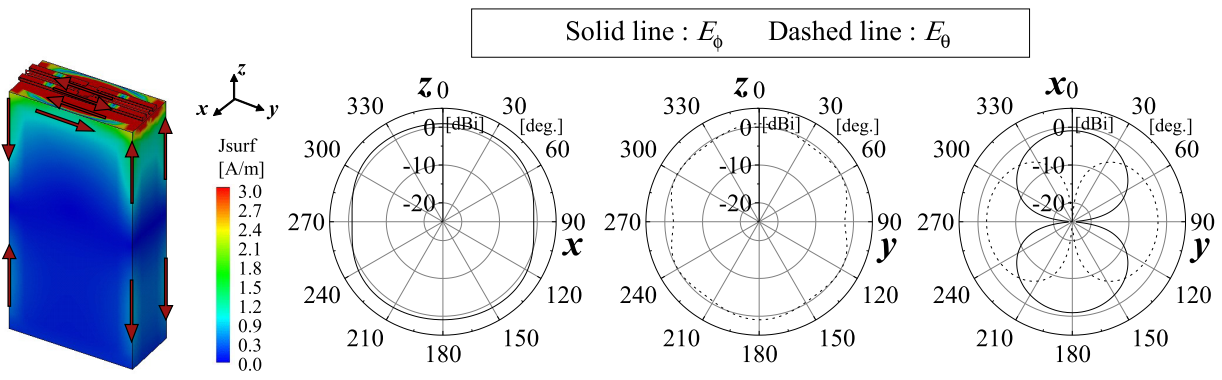

(c)

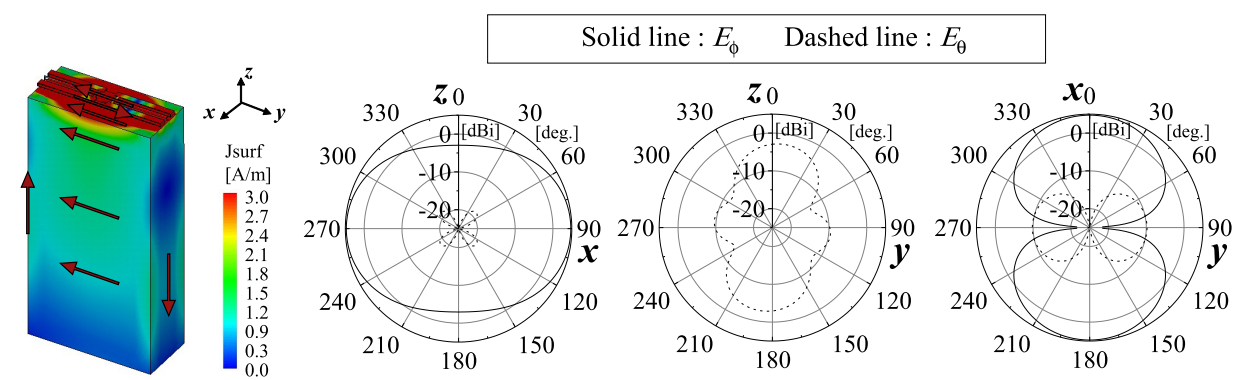

(d)

Fig. 2. (a) Analysis model. (b) Modal weighting coefficient. (c) Current distribution and radiation pattern of mode 2. (d) Current distribution and radiation pattern of mode 5 .

that toward the $-z$-axis. The maximum actual gain is $2.0 \mathrm{dBi}$. Figure $3 \mathrm{~d}$ shows the simulated radiation efficiency and total efficiency for each condition. In free space, the BFDA has a high input impedance and does not match the $50 \Omega$ feed line. When the BFDA is close to the infinite ground plane, the radiation efficiency is lower than that in free space, owing to a decrease in radiation resistance. When the BFDA is mounted on the metal casing, the surface current on the metal casing contributes to the radiation, and the radiation efficiency and total efficiency are improved compared with those on the infinite ground plane.

\section{Conclusion}

This study showed the excitation of the metal casing using the BFDA. The radiation was mainly radiated from the casing with the BFDA in close proximity, and the radiation from the BFDA was suppressed by its proximity to the ground plane. The radiation was based on the characteristic mode of the casing and was considered to 


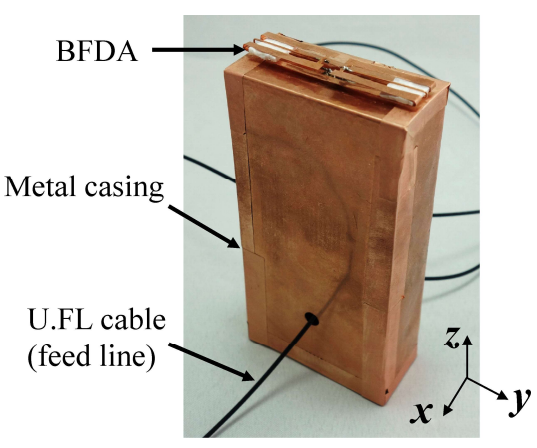

(a)

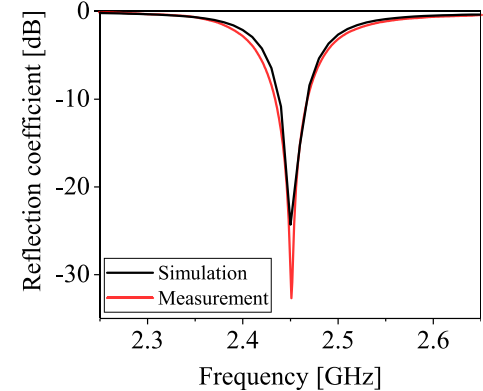

(b)

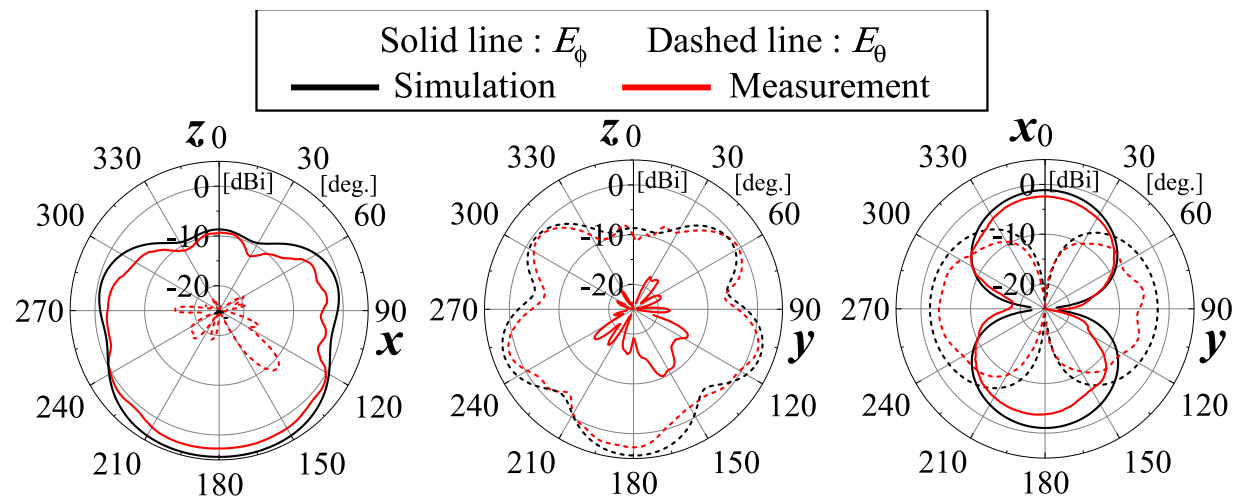

(c)

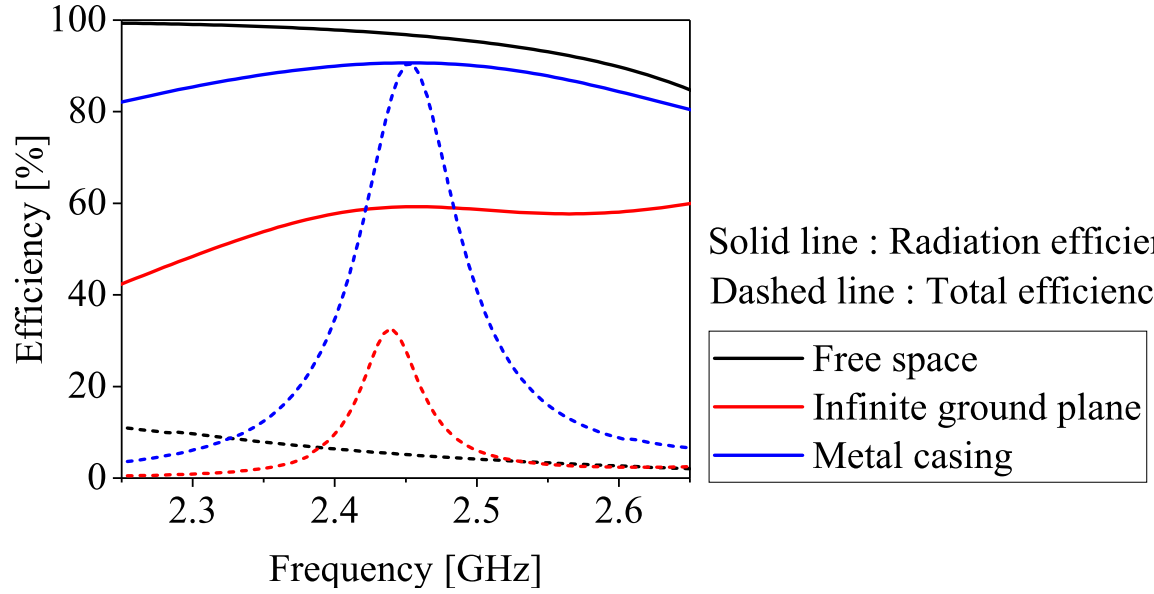

(d)

Fig. 3. (a) Fabricated antenna. (b) Reflection coefficient. (c) Radiation patterns. (d) Radiation efficiency (simulation).

be excited by the modified folded dipole antenna. In future work, the bandwidth will be expanded, and the excitation element will be miniaturized. 